\title{
OPEN Loss of Caveolin-1 and caveolae leads to increased cardiac cell stiffness and functional decline of the adult zebrafish heart
}

\author{
Dimitrios Grivas ${ }^{1,2}$, Álvaro González-Rajal| ${ }^{1,3}$, Carlos Guerrero Rodríguez ${ }^{4}$, Ricardo Garcia ${ }^{4}$ \& \\ José Luis de la Pompa ${ }^{1,2 \bowtie}$ \\ Caveolin-1 is the main structural protein of caveolae, small membrane invaginations involved in \\ signal transduction and mechanoprotection. Here, we generated cav1-KO zebrafish lacking Cav1 and \\ caveolae, and investigated the impact of this loss on adult heart function and response to cryoinjury. \\ We found that cardiac function was impaired in adult cav1-KO fish, which showed a significantly \\ decreased ejection fraction and heart rate. Using atomic force microscopy, we detected an increase \\ in the stiffness of epicardial cells and cells of the cortical zone lacking Cav1/caveolae. This loss of \\ cardiac elasticity might explain the decreased cardiac contraction and function. Surprisingly, cav1-KO \\ mutants were able to regenerate their heart after a cryoinjury but showed a transient decrease in \\ cardiomyocyte proliferation.
}

Caveolae are small membrane invaginations present in endothelial cells, fibroblasts and less abundantly, in cardiomyocytes $^{1-4}$. Caveolin-1 (Cav1) is the main structural protein of the caveolae ${ }^{5}$, as Cav1 deletion in mice diminishes caveolae formation ${ }^{6-8}$. Similarly, the caveolae associated protein Cavin1, is essential for caveolae formation, because its genetic deletion leads to loss of caveolae ${ }^{9}$. Caveolae participate in multiple cellular processes, including lipid homoeostasis and signal transduction ${ }^{1,10,11}$. In particular, Cav1 interacts directly with Transforming growth factor $\beta$ receptor-1 (TGF $\beta R 1$ ), blocking Smad complex nuclear translocation and, consequently, inhibiting transcriptional activation ${ }^{12}$. Furthermore, caveolae are involved in mechanoprotection, as they deliver the extra membrane needed for cells to buffer mechanical forces through rapid disassembly and flattening ${ }^{13,14}$. Physiologically, caveolae protect mouse cardiac endothelial cells from rupture caused by increased cardiac output ${ }^{10}$. Likewise, caveolae safeguard zebrafish skeletal muscle cells from rupture after vigorous activity ${ }^{15}$ and maintain notochord's integrity ${ }^{16,17}$.

Genetic inactivation of Cav1 in the mouse results in cardiac remodelling. Right ventricle dilatation and left ventricle hypertrophy are among the various cardiac defects associated with loss of caveolae ${ }^{1,8,18}$. Additionally, Cav1 mutant mice show defective heart function, including decreased systolic and diastolic function ${ }^{1,8,18,19}$, which is exacerbated after myocardial infarction ${ }^{20,21}$. Cardiac insult in Cav1 mutant mice also leads to aberrant fibrosis, mediated by increased Smad2/3 phosphorylation and macrophages infiltration ${ }^{21,22}$. In zebrafish, the cav1 gene generates two protein-coding transcripts, cav1a and cav1b, with the Cav1b protein being shorter, as it lacks the first 31 amino acids ${ }^{23}$. Resection of the ventricular apex in hearts of cav1a mutant zebrafish leads to regeneration defects 30 days post amputation (dpa), because of decreased cardiomyocyte proliferation and increased fibrosis in the amputation plain ${ }^{24}$. In addition, inactivation of both cav1a and cav $1 b$ transcripts, results in regeneration defects after ventricular resection only in heterozygous animals ${ }^{24}$.

Here, we have generated cav1-KO zebrafish and investigated the importance of Cav1 and caveolae in the mechanical properties of the cardiac tissue and in regeneration. We used the cryoinjury model of heart regeneration that leads to extensive fibrotic response, since Cav1 regulates negatively TGF $\beta$ pathway ${ }^{12}$. We found

${ }^{1}$ Intercellular Signalling in Cardiovascular Development and Disease Laboratory, Centro Nacional de Investigaciones Cardiovasculares Carlos III (CNIC), Melchor Fernández Almagro 3, 28029 Madrid, Spain. ${ }^{2}$ Ciber de Enfermedades Cardiovasculares, 28029 Madrid, Spain. ${ }^{3}$ Cell Division Lab, ANZAC Research Institute, Gate 3, Hospital Road, Concord, NSW 2139, Australia. ${ }^{4}$ Materials Science Factory, Instituto de Ciencia de Materiales de Madrid (ICMM), CSIC, 28049 Madrid, Spain. ${ }^{\circledR}$ email: jlpompa@cnic.es 
that while the absence of Cav1 does not affect cardiac regeneration, cav1-KO hearts show a transient decrease in cardiomyocyte proliferation during this process. Using atomic force microscopy (AFM)-force spectroscopy measurements ${ }^{25}$, we detected a substantial reduction in cardiac elasticity in cav1-KO animals. Accordingly, epicardial cells and cells of the cortical zone in cav1-KO hearts lacking caveolae are stiffer than wild type (WT) counterparts. Furthermore, cav1-KO hearts showed a severe ventricular dysfunction, underscoring the role of caveolae in the mechanical properties and homeostasis of the heart.

\section{Results}

Caveolin-1 expression in the intact and regenerating zebrafish heart. We began our analysis by examining Cav1 expression in intact hearts. We used the $\operatorname{Tg}\left(\right.$ fli1a:GFP) line ${ }^{26}$, which expresses GFP in the endocardium and endothelium, and stained with antibodies against Cav1 and tropomyosin (Fig. 1a). Robust Cav1 expression was detected in the vasculature (asterisks in Fig. $1 \mathrm{~b}, \mathrm{~b}^{\prime}, \mathrm{b}^{\prime \prime}$ ) and in the endocardium (arrowheads in Fig. $1 b, b^{\prime}, b^{\prime \prime}$ and $c, c^{\prime}, c^{\prime \prime}$ ). Strong expression was also found in the epicardium (arrows in Fig. 1b, $b^{\prime}, b^{\prime \prime}$ ), in the bulbus arteriosus and in the valves (Fig. 1a). Additionally, Cav1 expression was detected in the area between the cortical and trabecular myocardium (dashed area in Fig. 1b, b', b" and inset in Fig. 1b'). We then analysed Cav1 expression in the regenerating zebrafish heart after cryoinjury (Fig. 1d). We used the $T g(w t 1 b: G F P)$ line that expresses GFP in the epicardium upon injury ${ }^{27}$. Seven days post cryoinjury (dpci), Cav1 was strongly expressed in epicardial cells (Fig. 1e, $\mathrm{f}, \mathrm{f}^{\prime}$ brackets) covering the injured site, overlapping with GFP. High expression was also detected in the endocardium within the injured area (Fig. 1f, arrows). To confirm these observations, we utilised the $\mathrm{Tg}(f l i 1 a: G F P)$ line and found that Cav1 was expressed in $\mathrm{GFP}^{+}$endocardial cells invading the damaged tissue (Fig. 1g, h, h', arrows). We also surveyed the expression of caveolae-related genes during heart regeneration by quantitative (q)PCR (Fig. 1i). cav1 and cavin $1 b$ were upregulated after injury, in contrast to cav 2 and cav3 whose expression remained stable. These results show that Cav1 is expressed in the endocardium, endothelium and epicardium of the intact heart, three cell types that are activated during regeneration ${ }^{28-31}$. Also, upon injury, Cav1 expression is strongly increased in epicardial cells surrounding the injured site, and in the endocardium invading the injured area.

Generation of cav1-KO zebrafish by CRISPR/Cas9 editing. We generated cav1-KO zebrafish to study the role of Cav1 and caveolae in heart homeostasis and regeneration. We used CRISPR/Cas9 editing to target the third exon of cav1, which corresponds to the C-terminal region of the protein (Fig. 2a). The zebrafish cav1 gene generates two transcripts-cavla and cav1b-sharing the majority of the coding sequences, with cav $1 a$ giving rise to 31 amino acids longer protein ${ }^{23}$. We were able to introduce and identify mutations in the cav 1 locus, with the majority of them being small deletions (Fig. 2b). The predicted effect on the protein was an open reading frame shift that would lead to an amino acid change and the generation of a premature stop codon (Fig. 2c). We selected the cav $1^{c n 100}$ mutation, which showed significantly decreased cav1 expression, increased cav2 expression, but with no effect on cav3, cavin $1 b$ or cavy transcription (Fig. 2d). Mutant embryos had no morphological abnormalities, developed normally and were fertile (data not shown). We next investigated Cav1 expression by labelling 7 dpci WT and mutant samples with an antibody against Cavla (Fig. 2e-j and Supplementary Fig. S1a). The strong Cavla signal in the epicardium, endocardium, endothelium, bulbus arteriosus and valves (Fig. 2e-g) was lost in cav $1^{\text {cn } 100}$ hearts (Fig. $2 \mathrm{~h}-\mathrm{j}$ ). We repeated this analysis with an antibody that recognises both Cav1 proteins, Cavla and Cav1b (Fig. 2k-p and Supplementary Fig. S1b). Normal Cav1 expression in epicardium, endocardium and endothelium (Fig. $2 \mathrm{k}-\mathrm{m}$ ) was absent in cav $1^{\text {cn } 100}$ mutants (Fig. $2 \mathrm{n}-\mathrm{p}$ ), indicating the lossof-function (LOF) nature of the mutation. Cavin1 is also an essential component of caveola ${ }^{32}$ and deletion of Cav1 diminishes Cavin1 expression in mice ${ }^{33}$. We therefore asked whether Cavin1 was affected by Cav1 loss. Examination of $7 \mathrm{dpci}$ cav $1^{+/+}$cryoinjured hearts revealed strong Cavin 1 expression in the epicardium, in cardiomyocytes adjacent to the injured area, in the bulbus arteriosus and in the valves (Supplementary Fig. S2). Cavin1 expression was decreased in cav1 ${ }^{c n 100}$ hearts (Supplementary Fig. S2). Specifically, Cavin1 was absent in the valves, whereas its expression was greatly reduced in the bulbus arteriosus (Supplementary Fig. S2e) and in cardiomyocytes within the proliferative zone (Supplementary Fig. S2g, g'). Taken together, these results show that the cav $1^{\text {cn } 100}$ mutation leads to the loss of Cav1 and to the reduced expression of Cavin 1 in cryoinjured hearts.

Loss of caveolae in cav1 $\mathbf{1}^{c n 100}$ mutant hearts. Expression of Cav1 in a system without endogenous Cav1 expression leads to de novo caveolae formation ${ }^{5}$, whereas deletion of Cav1 results in loss of caveolae ${ }^{6-8}$. Hence, we asked whether cav $1^{\text {cn100 }}$ mutants could form caveolae. To address this, we analysed $\operatorname{cav} 1^{+/+}$and $\operatorname{cav} 1^{\text {cn } 100}$ hearts by transmission electron microscopy (TEM) (Fig. 3). We found caveolae in abundance in $c a v 1^{+/+}$hearts (Fig. 3a, $\mathrm{a}^{\prime}$ ), and the membrane of the endothelial cells was packed with caveolae-invaginations (Fig. $3 \mathrm{a}^{\prime}$, arrowheads). By contrast, $\operatorname{cav} 1^{\text {cn } 100}$ hearts were deprived of caveolae in the coronary vasculature of the cortical layer (Fig. 3b, b',c). No membrane-bound caveolae were detected, indicating the complete loss of caveolae in $c a v 1^{\text {cn } 100}$ mutant hearts.

Response of caveolae-depleted hearts to injury. We next examined the effects of caveolae loss in adult heart regeneration. We cryoinjured $c a v 1^{+/+}$and cav $1^{c n 100}$ hearts and allowed them to regenerate for 90 days. We then analysed the hearts by Acid Fuchsin Orange-G (AFOG) staining, which labels both the damaged area and the healthy myocardium. We found that homozygous cav $1^{\text {cn100 }}$ and heterozygous cav $1^{+/ c n 100}$ hearts had regenerated similarly to $c a v 1^{+/+}$hearts $90 \mathrm{dpci}$ (Fig. $4 \mathrm{~g}-\mathrm{j}$ ). Cryoinjury results in the formation of a scar tissue that progressively degrades during the course of 90 days ${ }^{34-36}$. Thus, we monitored the regeneration process by analysing injured hearts at 30 and $60 \mathrm{dpci}($ Fig. $4 \mathrm{a}-\mathrm{f})$. cav1 $1^{\text {cn } 100}$ hearts had a similar scar size to that of $\operatorname{cav} 1^{+/+}$ controls, both at 30 and $60 \mathrm{dpci}$. 

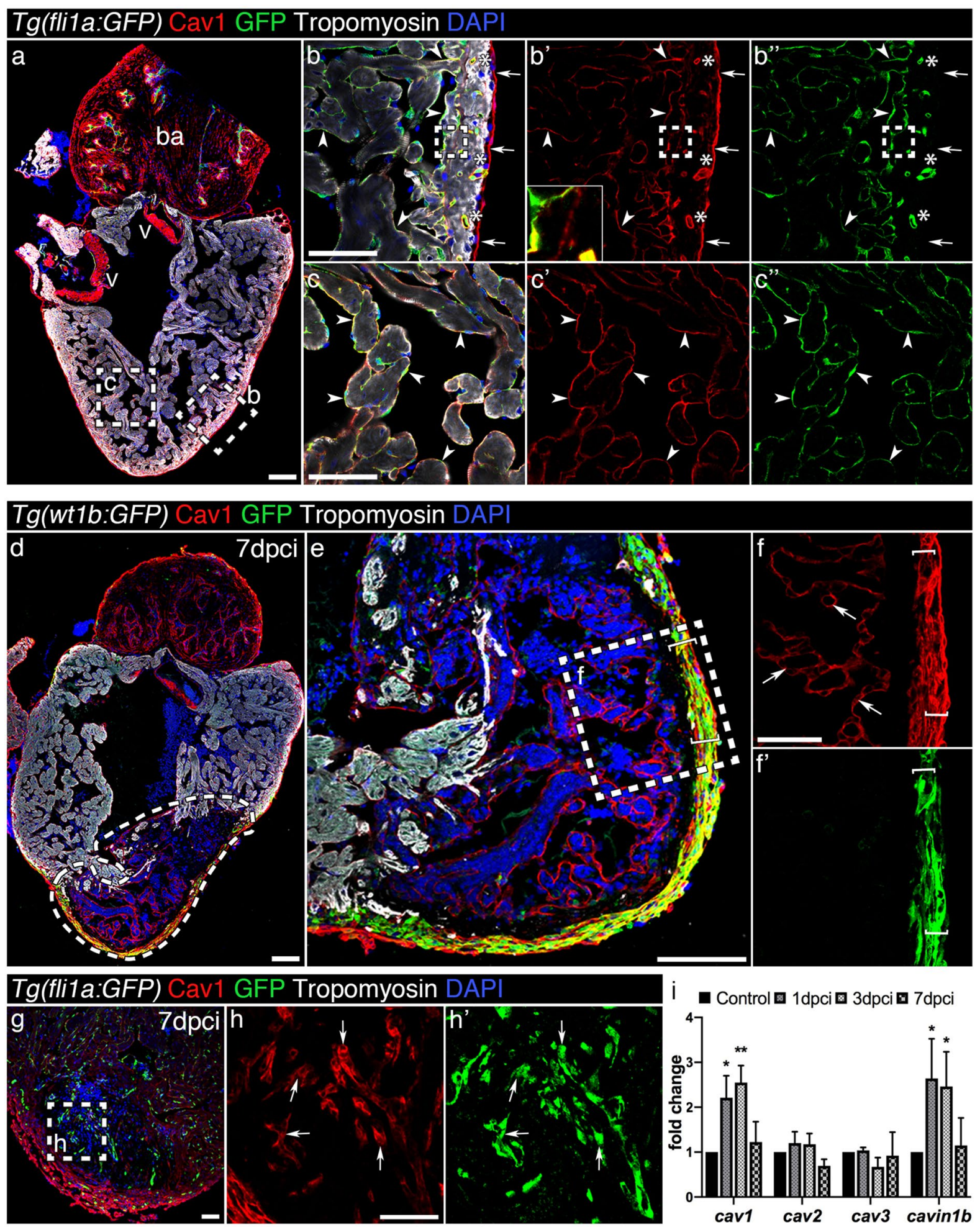

Figure 1. Caveolin-1 is expressed in the endothelium, endocardium and epicardium of the intact and injured adult zebrafish heart. (a) Immunofluorescence staining of Cav1 and Tropomyosin (cardiomyocytes) in an intact $\mathrm{Tg}(f l i 1 a$ :GFP) heart. ba, bulbus arteriosus; v, valves. (b-c'") Cav1 immunoreactivity in the epicardium (arrows) overlaps with GFP in the endothelium (asterisks) and endocardium (arrowheads). Cav1 is also expressed in the zone between the cortical and trabecular cardiomyocytes (insert in $\left.\mathbf{b}^{\prime}\right)$. (d-f $\left.\mathbf{f}^{\prime}\right)$ Cavl immunostaining in $7 \mathrm{dpci} \operatorname{Tg}($ wt 1 b:GFP) heart. The dashed area in (d) marks the injured area; Cav1 is expressed in the activated epicardium (e-f' brackets) and endocardium (f, arrows) upon injury. (g-h') Immunolabelling of Cav1 in a 7 dpci $T g(f l i 1 a: G F P)$ heart. (h-h') Arrows indicate Cav1 ${ }^{+}$endocardial cells. Scale bars: $100 \mu \mathrm{m}$ in (a), (d), (e) and $50 \mu \mathrm{m}$ in other panels. (i) qPCR analysis of caveolae-related genes during regeneration. Mean \pm s.d., BrownForsythe and Welch ANOVA tests, ${ }^{\star} P<0.05,{ }^{\star *} P<0.01$. 
a

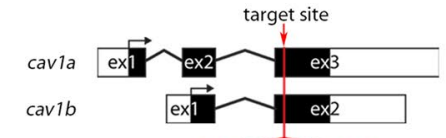

b

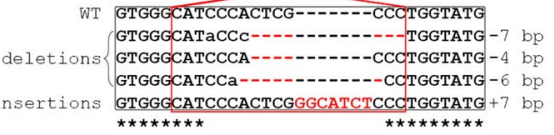

C

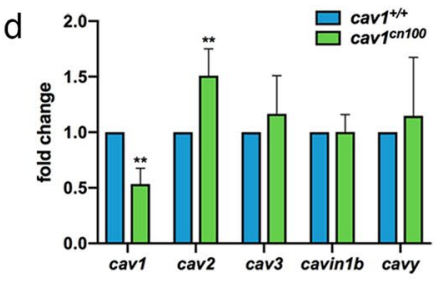

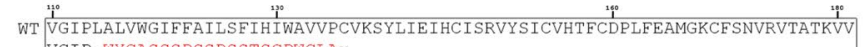
en100 VGIPPHYGASSSPSPPSSTSGPECLAX

VGIP--LVWGIFFA LLSEIHIWAVVPCVKSYLIEIHCISRVYSICVHTFCDPLEEAMGKCFSNVRVTATKVV

cn101 VGIPLGHLPGMGHLIRHPLLHPHLGRGALREELPNRDPLHQSSLIHLCAHLIRPTLX
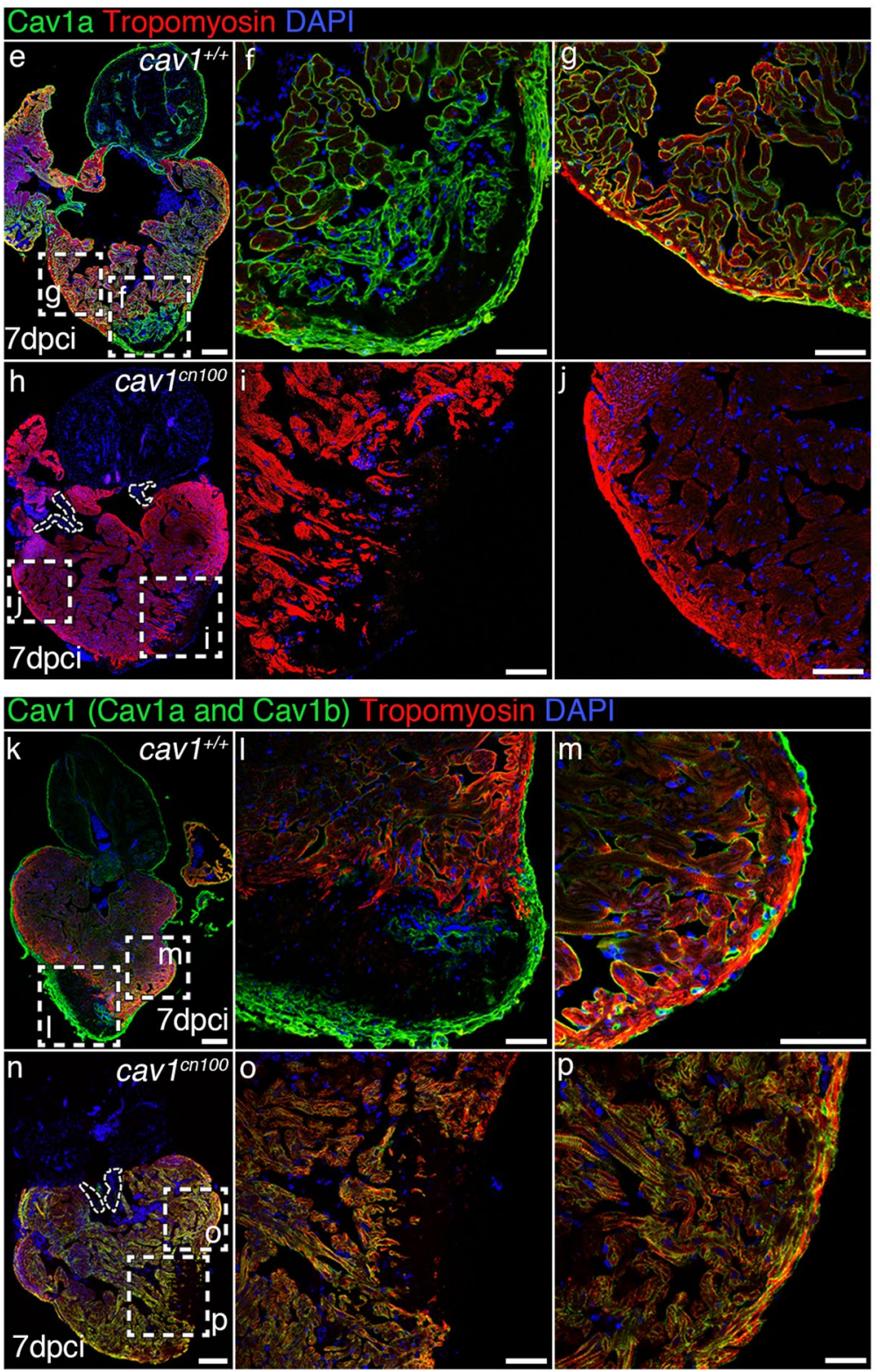

Figure 2. Generation of cav1-KO by CRISPR/Cas9 editing and transcriptional analysis. (a) Schematic representations of the two cav1 transcripts-cav1a and cav1b. Target site of the guide RNA is indicated by a red arrowhead. ex, exon. (b) Identified genetic mutations. Red dashed lines indicate deletions, red uppercase letters insertions, and black lowercase letters silent mutations. bp, base pairs. (c) Cav1 domain organisation and the predicted effect of the mutations on the protein. The novel amino acids are in red, $\mathrm{x}$ indicates a stop codon. (d) qPCR analysis of caveolae-related genes in two-day post fertilisation embryos. Mean \pm s.d., $\mathrm{t}$-test, ${ }^{* *} P<0.01$. (ej) Immunostaining of Cavla in 7 dpci hearts. $(\mathbf{f}, \mathbf{g}, \mathbf{i}, \mathbf{j})$ Higher magnification of the dashed boxes in $(\mathbf{e})$ and $(\mathbf{h})$, respectively. (k-p) Cav1 (Cav1 and Cav1b) immunolabelling of 7 dpci hearts. (1, m, o, p) Higher magnification of the dashed boxes in (k) and (n), respectively. Dotted areas in (h) and (n) mark the valves in the mutants. Scale bars: $100 \mu \mathrm{m}$ in (e), (h), (k), (n); $50 \mu \mathrm{m}$ in other panels. 
cav $1^{+/+}$

cav1 $1^{\mathrm{cn} 100}$
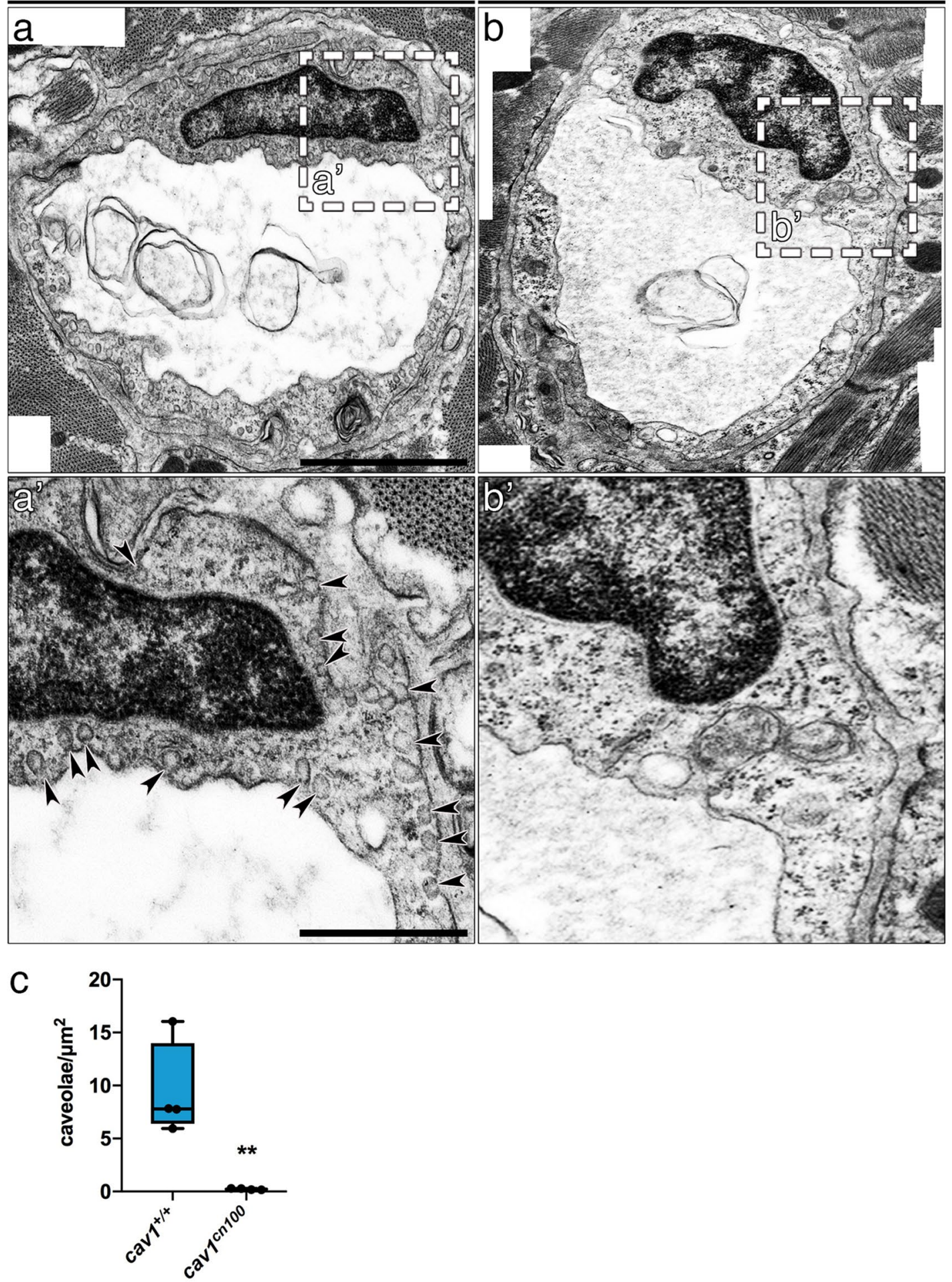

Figure 3. Loss of caveolae in cav1 $1^{c n 100}$ hearts. TEM images of $c a v 1^{+/+}\left(\mathbf{a}, \mathbf{a}^{\prime}\right)$ and $\operatorname{cav} 1^{c n 100}\left(\mathbf{b}, \mathbf{b}^{\prime}\right)$ endothelial cells from the coronary vasculature. $\left(\mathbf{a}^{\prime}, \mathbf{b}^{\prime}\right)$ Higher magnification of the dashed areas in (a) and (b). Arrowheads indicate membrane-bound caveolae. Scale bars: $1 \mu \mathrm{m}$ in (a), (b); $0.5 \mu \mathrm{m}$ in $\left(\mathbf{a}^{\prime}\right),\left(\mathbf{b}^{\prime}\right)$. (c) Caveolae number per $\mu \mathrm{m}^{2}$ of endothelial cell. $\mathrm{n}_{\mathrm{WT}}=\mathrm{n}_{\mathrm{cn} 100}=4$, t-test, ${ }^{* \star} P<0.01$.

To further investigate the effect of caveolae loss in heart regeneration, we examined another cav1 mutant, cav $1^{c n 101}$. Interestingly, expression of cav1 transcript in the cav $1^{\text {c101 }}$ mutant embryos was unchanged, whereas cav 2 was upregulated (Supplementary Fig. S3a), in accord with our observations in $\operatorname{cav} 1^{\text {cn100 }}$ mutants. In addition, 

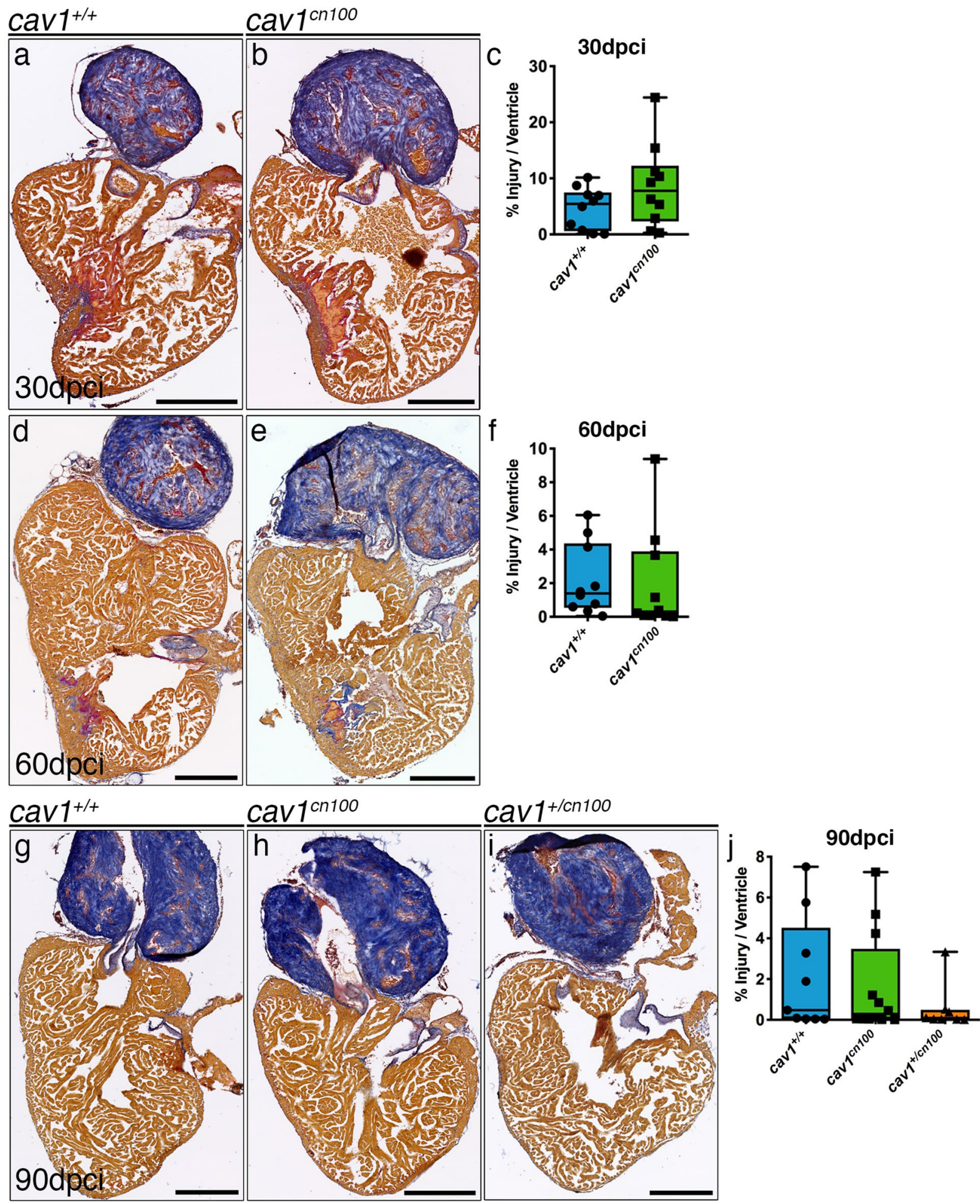

Figure 4. Heart regeneration is unaffected in $\operatorname{cav} 1^{c n 100}$ mutants. (a-j) $c a v 1^{+/+}$and $\operatorname{cav} 1^{c n 100}$ hearts were cryoinjured and harvested $30(\mathbf{a}, \mathbf{b}), 60(\mathbf{d}, \mathbf{e})$ or $90 \mathrm{dpci}(\mathbf{g}, \mathbf{h})$, and processed for AFOG staining, which labels collagen in blue, fibrin in red and healthy myocardium in brown. (i) heterozygous cav1 ${ }^{+/ c n 100}$ heart, $90 \mathrm{dpci}$. (c, $\mathbf{f}, \mathbf{j}$ ) Quantification of the injury site. Injuries were quantified as a percentage of the damaged tissue (collagen and fibrin) to the total area of the ventricle. $30 \mathrm{dpci}_{\mathrm{WT}}=\mathrm{n}_{\mathrm{cn} 100}=10 ; 60 \mathrm{dpci} \mathrm{n}_{\mathrm{WT}}=\mathrm{n}_{\mathrm{cn} 100}=10 ; 90 \mathrm{dpci} \mathrm{n}_{\mathrm{WT}}=9$, $\mathrm{n}_{\mathrm{cn} 100}=12$, $\mathrm{t}$-test. Scale bars $250 \mu \mathrm{m}$. 
the $\operatorname{cav} 1^{\text {cn101 }}$ mutation had the same effect on protein expression as the cav $1^{\text {cn100 }}$ mutation, with a loss of Cav1 expression (Supplementary Fig. S3). Likewise, caveolae were absent in cav ${ }^{\text {cn101 }}$ hearts, similar to our observations for $\operatorname{cav} 1^{\text {cn100 }}$ (Supplementary Fig. S4). We then cryoinjured cav $1^{+/+}$and $c a v 1^{c n 101}$ hearts and examined the regeneration process every 30 days for 90 days (Supplementary Fig. S5). cav ${ }^{c n 101}$ hearts regenerated normally and we did not detect any differences in the size of the injury at 30, 60 or $90 \mathrm{dpci}$. These results demonstrate that loss of Cav1 and caveolae do not affect heart regeneration.

Activation of TGF $\beta$ signalling and fibrosis are unaffected in cav1 ${ }^{c n 100}$ hearts. Caveolae are involved via Cav1 in the regulation of the TGF $\beta$ pathway ${ }^{12}$, a major signalling pathway that controls extracellular matrix (ECM) deposition, and is activated during zebrafish heart regeneration ${ }^{37}$. As both cav $1^{\text {cn100 }}$ and cav $1^{\text {cn101 }}$ hearts regenerated normally, we focused only on $\operatorname{cav} 1^{\text {cn100 }}$. To address TGF $\beta$ activity in $\operatorname{cav} 1^{\text {cn100 }}$ hearts upon cryoinjury, we quantified the nuclear localization of phospho-Smad3 (psmad3), a downstream effector of TGF $\beta$ (Supplementary Fig. S6a, b). We used 14 dpci $\operatorname{Tg}\left(f l i 1 a: G F P\right.$ ) hearts to calculate the proportion of psmad $3^{+}$nuclei in endocardial cells within the damaged tissue (Supplementary Fig. S6a', b', c) and in the cardiomyocytes surrounding the injured site (Supplementary Fig. S6a", b", d). Analysis revealed that TGF $\beta$ signalling was equally active in control and cav $1^{\text {cn100 }}$ hearts, in both endocardial cells and cardiomyocytes (Supplementary Fig. S6c, d). Heart cryoinjury in the Cav1-KO mouse leads to extensive collagen deposition and cardiac remodelling ${ }^{22}$. Thus, we exploited the AFOG staining protocol to examine the collagen and fibrin content after injury, and we also measured ventricular size (Supplementary Fig. S7a, b). We found no differences between cav1 $1^{+/+}$and $\operatorname{cav} 1^{\text {cn100 }}$ hearts, neither in collagen deposition nor in fibrin amount in the injury, nor in the size of the ventricle. Furthermore, because hearts of Cav1-KO mice show increased interstitial fibrosis ${ }^{1,6,18,38}$, we examined this parameter in intact $c a v 1^{c n 100}$ hearts using Picrosirius Red to stain collagen fibres. Results showed no difference in interstitial fibrosis between $c a v 1^{+/+}$and $c a v 1^{\text {cn100 }}$ hearts (Supplementary Fig. S7c-e). Thus, loss of Cav1 and caveolae does not affect TGF $\beta$ activity or fibrosis in intact or cryoinjured hearts.

Epicardial, endocardial and cardiomyocyte function in cav1 $1^{c n 100}$ hearts upon injury. We next examined the behaviour of the different cell types involved in the regeneration process. We first analysed the epicardium and endocardium, where Cav1 is highly expressed 7 dpci (Supplementary Fig. S8). We crossed the $\mathrm{Tg}(w t 1 b: G F P)$ line to $c a v 1^{c n 100}$ to study epicardial proliferation and we found no difference in epicardial proliferation between $c a v 1^{+/+}$and $c a v 1^{\text {cn100 }}$ hearts (Supplementary Fig. S8a, b, c). We then evaluated the abundance of endocardial cells within the damaged tissue by crossing double transgenic fish $T g(f l i 1 a: G F P) / T g(m y l 7: m R F P)$ expressing GFP in endocardial/endothelial cells and RFP in the membrane of cardiomyocytes, with cav1 $1^{\text {cn100 }}$ mutants (Supplementary Fig. S8d, e). Three-dimensional volume rendering and analysis of the $\mathrm{GFP}^{+}$cells inside the RFP- area showed that endocardial cells in cav1 ${ }^{c n 100}$ hearts populated the injured area similarly to those of cav $1^{+/+}$hearts (Supplementary Fig. S8f). These data indicate that epicardial proliferation and the behaviour of endocardial cells are unchanged in cav $1^{\text {cn100 }}$ injured hearts.

Cardiomyocyte proliferation has been reported to be reduced upon ventricular resection in a cavla-KO zebrafish model ${ }^{24}$. The cardiomyocytes adjacent to the injured area $7 \mathrm{dpci}$ are highly proliferative $\mathrm{e}^{39}$ and we analysed BrdU incorporation in control and $c a v 1^{c n 100}$ hearts (Fig. 5a, b, a', b'). Cardiomyocyte proliferation was significantly lower in $c a v 1^{c n 100}$ hearts than in $c a v 1^{+/+}$hearts (Fig. 5c). We confirmed this result in the cav1 $1^{\text {cn101 }}$ animals (Supplementary Fig. S9). We then addressed the proliferation status of cardiomyocytes at 14 dpci (Fig. $5 \mathrm{~d}, \mathrm{e}$ ). We found that at this time point, $\operatorname{cav} 1^{\text {cn100 }}$ cardiomyocytes proliferated at the same rate as $c a v 1^{+/+}$cardiomyocytes (Fig. 5d', e', f). These data indicate that loss of caveolae leads to the transient attenuation in cav $1^{\text {cn100 }}$ cardiomyocyte proliferation at $7 \mathrm{dpci}$, which is normalised by $14 \mathrm{dpci}$, leading to normal cardiac regeneration at $90 \mathrm{dpci}$.

Impaired heart function and cardiac elasticity in cav1 $1^{\text {100 }}$ mutants. Cav1-deficient mice have been reported to show decreased systolic function ${ }^{1,8}$. Therefore, we analysed heart function in our mutants using ultrasound imaging. Echocardiography of eleven months-old adult $c a v 1^{+/+}$and $\operatorname{cav} 1^{\text {cn } 100}$ animals revealed that cav $1^{\text {cn100 }}$ hearts were less efficient in pumping blood than control hearts as their ejection fraction was significantly lower (Fig. 6a). Additionally, the heart rate of $c a v 1^{c n 100}$ animals was lower than in control siblings (Fig. 6b). These findings indicate that caveolae are essential for normal cardiac function.

Caveolae provide protection against mechanical stress ${ }^{13,14}$. Because the biomechanical properties of cells influence the behaviour of tissues ${ }^{40}$ and caveolae are involved in mechanoprotection, we investigated the mechanical response of $\mathrm{cav1}^{+/+}$and $\operatorname{cav} 1^{c n 100}$ cardiac tissue to gain insight into the differences observed in cardiac function. We used AFM to determine the response of adult cav $1^{\text {cn100 }}$ hearts to force. Freshly isolated hearts were placed on an agarose gel, with the apex of the ventricle oriented towards the direction of the cantilever, and force measurements were taken (Fig. $6 \mathrm{c}-\mathrm{e}$ ). We found that cardiac tissue stiffness was significantly greater in cav $1^{\text {cn100 }}$ animals than in control animals (Fig. 6f). Applying the same force, the extent of deformation was 1.5 times greater in control hearts than in cav $1^{\text {cn } 100}$ hearts, without causing permanent deformation as the curves returned to zero (Fig. 6g). This result suggests that changes in epicardial and underlying cells, including cortical myocytes, were responsible for the observed difference in stiffness that we detected. That is because the experiments were performed by producing a compressive deformation (indentation) of $1.5 \mu$ min cav1 ${ }^{c n 100}$ mutant hearts. The force-distance curves (Fig. $6 \mathrm{~g}$ ) did not show evidence of tissue and/or cell rupture during the deformation. Therefore, the epicardium is a major contributor to the measured stiffness ( $550 \mathrm{~Pa}$ ). However, the use of large probes $(\mathrm{R}=30 \mu \mathrm{m})$ implies that deformation is also transmitted to surrounding regions beyond the $1.5 \mu \mathrm{m}$ indentation depth. By using bottom-effect corrections ${ }^{41}$ we estimate that the cells within 5-10 $\mu \mathrm{m}$ distance from the contact point will also contribute to the measured stiffness. Membrane invaginations such as caveolae, provide the necessary stretch capacity for cells to buffer the impact of mechanical forces ${ }^{14}$. Hence, the absence 

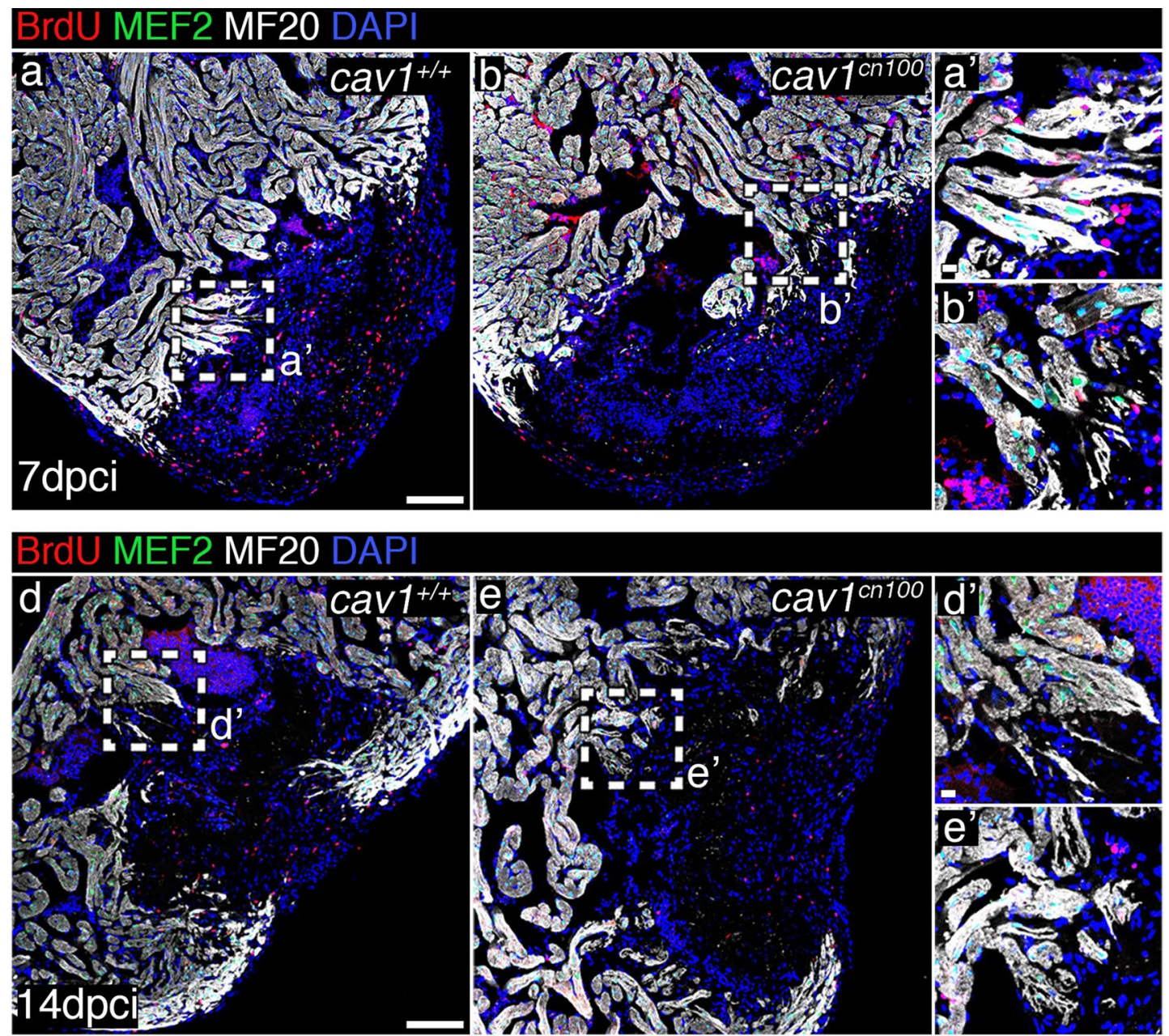

C

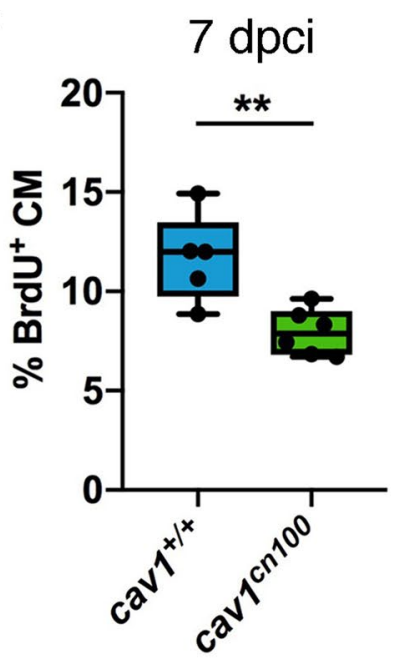

f

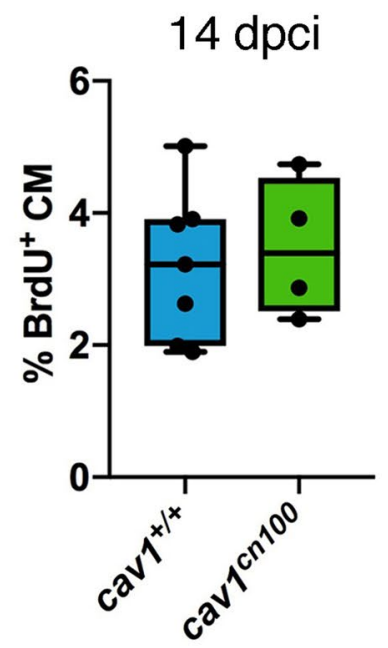

Figure 5. Cardiomyocyte proliferation is transiently reduced upon cryoinjury in $\operatorname{cav}^{c n 100}$ hearts. (a, b) Immunolabelling of $7 \mathrm{dpci} c a v 1^{+/+}$and $c a v 1^{c n 100}$ hearts for BrdU, MEF2 and MF20. (a', $\left.\mathbf{b}^{\prime}\right)$ Magnifications of the dashed areas in (a) and (b). (c) Cardiomyocyte proliferation rate was measured by quantifying the $\mathrm{BrdU}^{+} / \mathrm{MEF}^{+}$nuclei to the total cardiomyocyte number in a $100 \mu \mathrm{m}$ radius around the injured area. $\mathrm{CM}$, cardiomyocytes. $\mathrm{n}_{\mathrm{WT}}=5, \mathrm{n}_{\mathrm{cn} 100}=6$, t-test, ${ }^{\star *} P<0.01$. (d, e) Immunostaining of $14 \mathrm{dpci}$ cav $1^{+/+}$and cav1 ${ }^{\text {cn100 }}$ hearts for BrdU, MEF2 and MF20. (d' $\left.\mathbf{d}^{\prime}, \mathbf{e}^{\prime}\right)$ Magnifications of the dashed areas in (d) and (e). (f) Quantification of cardiomyocyte proliferation at 14 dpci. $\mathrm{n}_{\mathrm{WT}}=7, \mathrm{n}_{\mathrm{cn} 100}=4$, t-test. Scale bars: $100 \mu \mathrm{m}$ in (a), (b), (d), (e); $10 \mu \mathrm{m}$ in $\left(\mathbf{a}^{\prime}\right),\left(\mathbf{b}^{\prime}\right),\left(\mathbf{d}^{\prime}\right),\left(\mathbf{e}^{\prime}\right)$. 
a

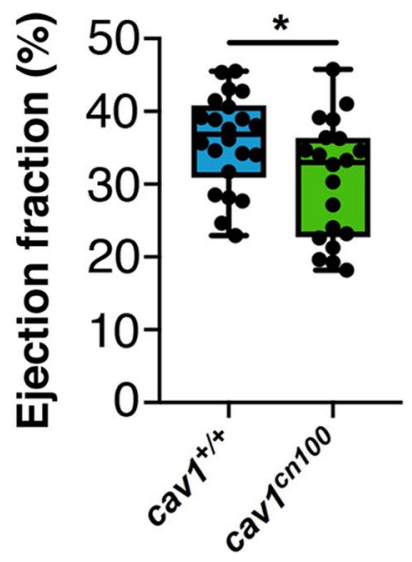

b

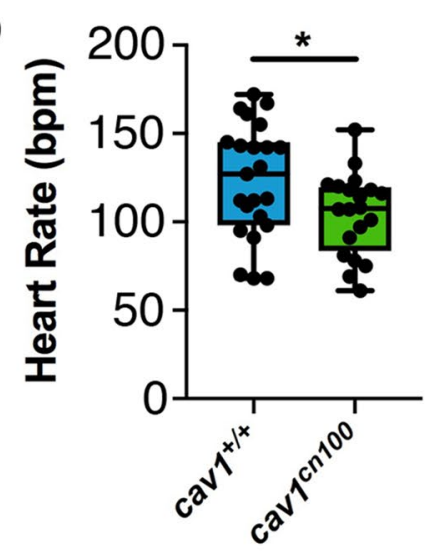

C

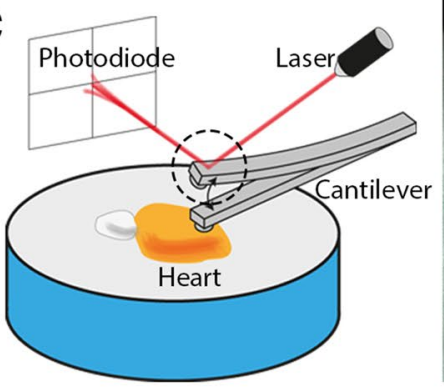

$f$

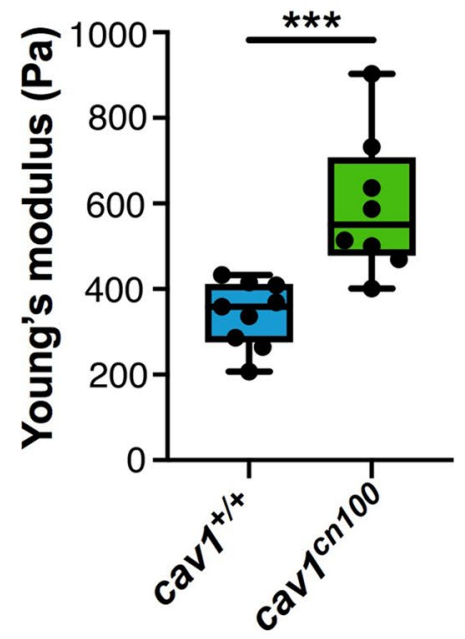

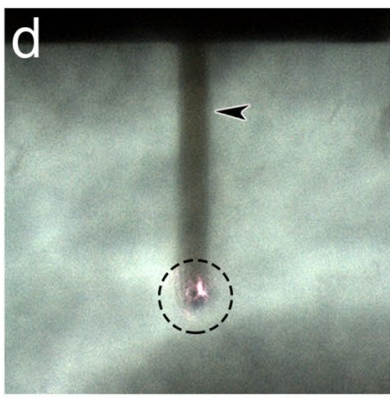

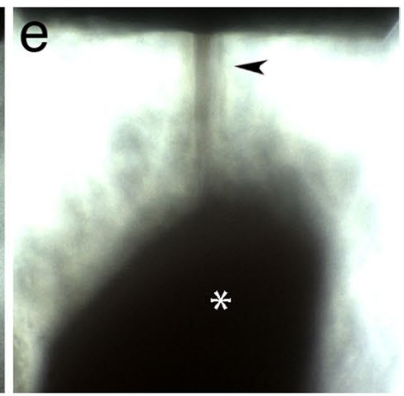

9

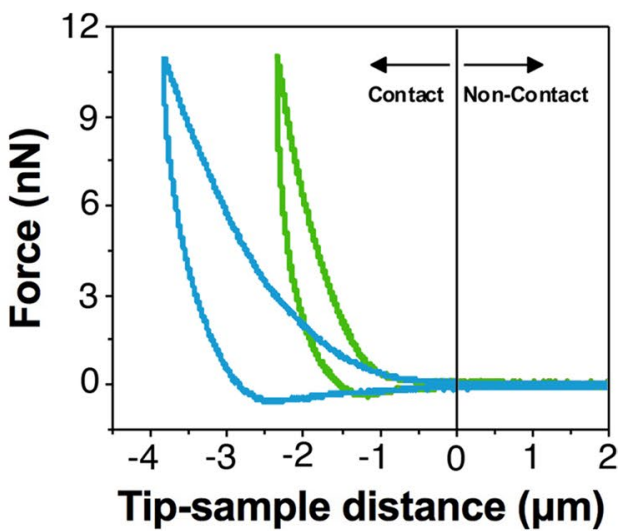

$\operatorname{cav1}^{+/+}$ cav1 100

Figure 6. Impaired cardiac function and stiffer heart tissue in caveolae-deprived cav $1^{\text {cn100 }}$ mutants. (a) Quantification of ejection fraction in $c a v 1^{+/+}$and cav $1^{c n 100}$ hearts. $\mathrm{n}_{\mathrm{WT}}=22, \mathrm{n}_{\mathrm{cn} 100}=20$. (b) Heart rate measurements in cav1 $^{+/+}$and cav1 ${ }^{c n 100}$ animals. $\mathrm{n}_{\mathrm{WT}}=23, \mathrm{n}_{\mathrm{cn} 100}=20$. t-test, ${ }^{\star} P<0.05$. (c) AFM set-up. Cartoon made with Adobe Illustrator CC2018 (www.adobe.com). (d, e) Images of the cantilever (arrowhead), the laser beam on top of the cantilever (dashed circle, also in c) and the ventricle (asterisk). Images were taken with an inverted optical microscope (AXIO Observer D1; Carl Zeiss, Germany, see Methods). (f) Biomechanical characterization of $c a v 1^{+/+}$and $c a v 1^{c n 100}$ ventricles as measured by AFM-force spectroscopy and expressed in Young's moduli. $\mathrm{n}_{\mathrm{WT}}=9, \mathrm{n}_{\mathrm{cn} 100}=8$, $\mathrm{t}$-test, ${ }^{* *} P<0.001$. (g) Force-distance graph for indentation and retraction of the cantilever over the ventricular surface. 
of membrane reservoirs, caveolae, due to the loss of the broad and robust expression of Cav1 in the ventricle, results in increased tissue stiffness.

\section{Discussion}

Caveolin-1 is the main structural protein of caveolae, small membrane invaginations involved in signal transduction and mechanoprotection ${ }^{7,13,14}$. Here, we investigated the importance of Cav1 and caveolae in heart homeostasis and regeneration after cryoinjury in zebrafish. We found that while Cav1 expression in the heart is strongly increased upon cryoinjury, its deletion, resulting in loss of caveolae, does not affect heart regeneration. However, loss of Cav1 and caveolae leads to increased stiffness of epicardial cell and cells of the cortical zone, including cardiomyocytes, and impaired cardiac function.

We found that Cav1 is expressed in the endothelium, endocardium and epicardium of intact adult hearts. Also, cav 1 transcription is upregulated following injury and Cav1 is strongly expressed 7 dpci in epicardial cells covering the damaged cardiac tissue, and in the endocardium invading the injured area. We generated cav1$K O$ zebrafish strains to examine the role of Cav1 in the heart. We used two cav1 mutant alleles, cav $1^{\text {cn } 100}$ and cav $1^{c n 101}$. Both mutations are LOF alleles, as they led to the lack of both Cavla and Cav1b proteins expression. TEM analysis showed that caveolae were completely absent in both cav $1^{c 100}$ and cav $1^{c 101}$ hearts, whereas they were abundant in $c a v 1^{+/+}$hearts. Thus, $c a v 1^{c n 100}$ and $c a v 1^{c n 101}$ mutations lead to loss of Cav1 and caveolae. This loss also affected Cavin 1 protein expression, although transcription was unaffected. The cav1-KO hearts lacked Cavin 1 in the valves and the overall expression of the protein was decreased, which is in accord with results from the Cav1-KO mouse ${ }^{32,33}$.

We investigated the effect of Cav1 and caveolae loss in cardiac regeneration using the cryoinjury model, as it mimics the mammalian myocardial infarction in formation of necrotic myocardium and scar tissue, which in the zebrafish gradually resolves leading to complete tissue regeneration at $90 \mathrm{dpci}^{34-36}$. We found that mutant hearts carrying the cav $1^{c n 100}$ or cav $1^{c n 101}$ LOF mutations in heterozygous or homozygous condition regenerate similar to the WT heart upon cryoinjury and we did not detect differences in injury size at 30 and 60 dpci. These findings were unexpected, as it has been reported that ventricular resection in heterozygous cav1-KO zebrafish results in enhanced fibrosis, leading to defects in heart regeneration $30 \mathrm{dpa}^{24}$. The fibrotic response in ventricular resection is minimum, whereas cryoinjury leads to massive fibrosis and to the formation of a transient scar tissue $^{34-36,42}$. However, our data indicate that the formation and resolution of the scar tissue is unaffected by Cav1 loss. Additionally, caveolin-1 negatively regulates TGF $\beta$ signalling ${ }^{12,]}$ and loss of murine CAV1 enhances collagen deposition after injury ${ }^{21,22}$. Notably, we found no changes in the quantity of collagen within the damaged tissue between cav1-KO and WT hearts, or in TGF $\beta$ pathway activation. Likewise, there were no signs of interstitial fibrosis in intact cav1-KO hearts, as has been reported in murine Cav1-KO models ${ }^{1,6,18,38}$. These results suggest activation of a compensatory mechanism ${ }^{43}$ that buffers Cav1 loss, which in heterozygosity might be induced stronger upon cryoinjury than after resection, in which fibrosis is minimal. Hence, since heart regeneration after ventricular resection lasts up to 60 days $^{42}$, it would be of interest to examine the process at the final time point. Intriguingly, $\operatorname{cav}^{c n 100}$ mutation results in decreased expression of cav1 transcript, whereas, cav 1 transcription is unchanged in $\operatorname{cav}^{c n 101}$. However, both of our mutations lead to loss of Cav1 protein expression. This could suggest that compensation might be triggered also after protein loss ${ }^{43}$, or that a Cav1-independent TGF $\beta$ regulatory pathway may exist in zebrafish heart regeneration.

We detected Cav1 in the epicardium covering the injured area and in endocardial cells invading the damaged tissue. Upon injury, the epicardium is also populated with fibroblasts and macrophages that contribute to fibrosis ${ }^{44,45}$, and loss of Cav1 in mice is associated with increased fibrosis and macrophage infiltration after cardiac injury ${ }^{21,22}$. The proliferation rate of epicardial cells was unchanged in cav1-KO hearts, indicating that these two cell types are unaffected in our model. In addition, endocardial cells have been associated with collagen deposition ${ }^{31}$ and Cav1 was completely lost in these cells. However, endocardial cell abundance was similar between WT and $c a v 1^{c n 100}$ hearts, suggesting that their proliferation and migration was unaffected. Collectively, these findings further indicate that loss of Cav1 and caveolae does not lead to deregulation of fibrosis in intact or in cryoinjured hearts. Moreover, loss of Cavl and caveolae triggered a transient but significant decrease in cardiomyocyte proliferation $7 \mathrm{dpci}$. This was not reflected in defective or even delayed regeneration. Indeed, cardiomyocyte proliferation in mutant fish $14 \mathrm{dpci}$ was similar to that of controls, suggesting that cardiomyocytes were overall able to increase in numbers, populate the damaged area and contribute to normal heart regeneration in cav1 mutants. In summary, considering that the heart of both of homozygous or heterozygous cav1 LOF mutants regenerates normally, we can only conclude that Cav1 and caveolae are not required for zebrafish heart regeneration.

Systolic and diastolic functions are also affected in Cav1-null mouse hearts ${ }^{1,8,18,19}$ and, in line with this finding, the $c a v 1^{c n 100}$ line showed impaired cardiac performance, as indicated by the reduced EF and a lower heart rate. As we did not detect cardiac fibrosis, the reduction in cardiac elasticity is a plausible explanation for the cardiac dysfunction in cav1-KO fish. Membrane invaginations such as caveolae provide the necessary stretch capacity for cells to buffer the impact of mechanical forces ${ }^{13,14}$. Our AFM-force spectroscopy analysis revealed that cav1-KO hearts were significantly stiffer than WT hearts, as cardiac tissue elasticity was decreased almost two-fold after loss of Cav1 and caveolae. The sensitivity of AFM allows us to conclude that the measurements represent the stiffness of both the epicardial cells and the underlying cortical cells, including cardiomyocytes. We have shown that Cav1 is strongly expressed in the epicardium of intact hearts and that its expression is lost in cav $1^{\text {cn } 100}$ hearts. Additionally, RNA-seq data show that cav1 expression is moderate in cortical cardiomyocytes, but significantly higher than in trabecular cardiomyocytes ${ }^{44}$. Caveolae are required for smooth muscle cell contractility ${ }^{46,47}$ and cell contraction results in changes to membrane tension that caveolae buffer ${ }^{14}$. In addition, Cav1 and caveolae regulate RhoA activation (a GTPase protein that regulates the cytoskeleton), which in turn regulates actomyosin 
contractility ${ }^{48-50}$. Accordingly, these data suggest that loss of Cav1 and caveolae impairs cell elasticity and/or contractility and, consequently, pumping efficiency, which could explain the significant reduction in cardiac performance. Cav1 deficiency in the mouse leads to diverse cardiac phenotypes that are attributed to extensive fibrosis and endothelial loss of Cav1 and caveolae. Our results demonstrate that loss of Cav1 and caveolae result in cardiac stiffening accompanied by reduced cardiac function, suggesting that a global change in the mechanical properties of the heart leads to the cardiac phenotypes observed in Cav1-deficiency models.

\section{Methods}

Zebrafish husbandry and transgenic lines. Animal studies were approved by the CNIC Animal Experimentation Ethics Committee and by the Community of Madrid (Ref. PROEX 83.8/20). Animal procedures conformed to EU Directive 2010/63EU and Recommendation 2007/526/EC regarding the protection of animals used for experimental and scientific purposes, enforced in Spanish law under Real Decreto 53/2013. Zebrafish were raised under standard conditions at $28^{\circ} \mathrm{C}$ as described ${ }^{51}$. Experiments were performed with 5-14-monthold adults.

CRISPR/Cas9 injections and nature of mutant alleles. We used the oligos cav1Fwd CACCGGTGG GCATCCCACTCGCCC and cav1Rvs AAACGGGCGAGTGGGATGCCCACC for the generation of cav1-KO zebrafish. The oligos were inserted into the pX330-U6-Chimeric_BB-CBh-hSpCas9 vector ${ }^{52}$, which was linearized with BbsI enzyme (New England Biolabs, Ipswich, MA). Primers with the T7 polymerase promoterspecific sequences, cav1 T7 Fwd TAATACGACTCACTATAGGTGGGCATCCCA and Rvs AAAAAGCACCGA CTCGGTGCCA, were used to amplify the guide RNA, which was injected into one-cell state embryos together with Cas9 protein (New England Biolabs). Mutant animals were identified by PCR using the primers: cav1Fwd GGCGAGCTTCACCACCTTC and cav1Rvs GCTCTTCACGCAAGGCACCA. Both mutant alleles generated and characterized in this study (cav1 $1^{\text {cn100 }}$ and $\left.\operatorname{cav1}^{\text {cn101}}\right)$ carry loss-of-function or KO mutations.

Adult heart cryoinjury. Fish were anesthetised by immersion in $0.04 \%$ tricaine (Sigma-Aldrich, St Louis, $\mathrm{MO}$ ) in fish water and placed on a wet sponge under a stereoscope with the ventral side exposed. The cardiac cavity was opened using microscissors and microforceps, and the pericardium was removed. The ventricle of the heart was exposed and dried and was then touched by a copper-made probe previously immersed in liquid nitrogen ${ }^{28}$. The fish were immediately returned to water to recover.

Bromodeoxyuridine injection. Adult fish were anesthetised and placed on a wet sponge under a stereoscope. 5-bromo-2'-deoxyuridine (BrdU) was diluted in phosphate-buffered saline (PBS) to $2.5 \mathrm{mg} / \mathrm{ml}$ and $30 \mu \mathrm{l}$ were injected intraperitoneally 24 -h before dissection of the hearts.

Echocardiography. Analysis of cardiac function by echocardiography in eleven months-old adult fish was performed as described ${ }^{53}$. Briefly, the fish were anesthetised by immersion in $60 \mathrm{mM}$ tricaine and $3 \mathrm{mM}$ isoflurane in fish water and transferred to a sponge immersed in the same solution. Images were acquired using the VEVO 2100 system (VisualSonics Inc., Toronto, ON, Canada) with a 50-MHz ultrasound probe. The transductor was immersed in the medium dorsally to the cardiac cavity. The fish were immediately transferred to fresh water to recover after the procedure.

Histological stains. Acid Fuchsin Orange G-staining (AFOG) and Picrosirius Red staining were performed following standard protocols ${ }^{42}$.

Immunofluorescence. Sections of paraffin-embedded tissue were permeabilised with PBT (PBS with $0.01 \%$ TritonX-100) and washed with PBS before incubation with blocking solution ( $2 \%$ bovine serum albumin, $10 \%$ goat serum and $2 \mathrm{mM} \mathrm{MgCl}_{2}$ in PBS). Sections were then incubated overnight at $4^{\circ} \mathrm{C}$ with the antibodies of choice. Caveolin 1a (Cavla), this isoform is 31 amino acids longer in the N-terminus region than Cavlb. The Cavla antibody (Cell Signalling Technology, catalogue \#D46G3) was raised against the N-terminus region of Cav1a, including residues surrounding Glu20. Caveolin-1 (BD Transduction Laboratories, catalogue \#610059), this antibody recognizes amino acids common to both Cavla and Cavlb, residues 1-97. PTRF/Cavin1 (Atlas Antibodies AB, Stockholm, Sweden, catalogue \#HPA049838), GFP (Aves Labs, Tigard, OR, catalogue \#GFP1010), MEF-2 (Santa Cruz Biotechnology, Santa Cruz, CA, catalogue \#sc-313), tropomyosin, MF-20 (MHC, myosin, sarcomere, DSHB, Iowa City, IA, USA), phosho-Smad3 (Abcam, Cambridge, MA, catalogue \#ab52903) and BrdU (BD Transduction Laboratories, catalogue \#347580). The following day, sections were incubated with the appropriate secondary antibody and mounted after DAPI staining.

Western blot (WB). Protein expression analysis by WB was performed in pools of three caudal fins. Samples were incubated in buffer $(150 \mathrm{mM} \mathrm{NaCl}, 25 \mathrm{mM}$ Tris pH 7.5, $1.5 \mathrm{mM} \mathrm{MgCl}, 1 \%$ Triton X-100, $10 \mathrm{mM}$ DTT, phosphatase and protease inhibitors) and sonicated (NESLAB RTE 7). Equal amount of proteins (30 $\mathrm{mg})$ were used for SDS-page electrophoresis. Proteins were transferred to PVDF Immobilon-P (Millipore) and blocked with 5\% milk. Primary antibodies against Cavla, Cav1 and alpha-Tubulin (Thermofisher scientific, catalogue \#62204) were diluted in TBS-Tween 0.1\%/2\% BSA and incubated overnight and then incubated with secondary antibody coupled to horseradish peroxidase (Dako Cytomation). Membranes were incubated with the Immobilon Western HRP substrate (Millipore) and imaged with the Image Quant LAS 4000 mini machine. 
Whole-mount confocal imaging. Analysis of endogenous fluorescence of whole-mount hearts was performed as described ${ }^{31}$. Briefly, hearts were fixed overnight with $2 \%$ paraformaldehyde and after several PBS washes the tissues were immersed in $3 \%$ agarose. Samples were then incubated in CUBIC I solution ${ }^{54}$ at $37^{\circ} \mathrm{C}$ for one week. Agarose blocks containing the hearts were mounted for imaging on a petri dish and approximately $700 \mu \mathrm{m}$ of the injured ventricle was scanned on a Leica SP8 confocal microscope (Leica Microsystems, Wetzler, Germany) using a $10 \times$ objective.

Quantitative RT-PCR. Three-to-five biological replicates with three technical replicates of each sample were used for the expression analysis of genes by qPCR using the power SYBR Green Master Mix (Applied Biosystems, Foster City, CA) and the ABI PRISM 7900HT FAST Real-Time PCR System. All measurements were normalised to the expression of $18 \mathrm{~s}^{55}$. The following primers were used for the qPCR analysis: 18 sFwd TCGCTA GTTGGCATCGTTTATG, $18 s$ Rvs CGGAGGTTCGAAGACGATCA, cav1Fwd TGGGATGGGGGAATGGAA AC, cav1Rvs TAAACGGCGAGTGAGCGTAT, cav2Fwd GCGTTTATTGCAGGGATTGT, cav2Rvs GGATCA CTGGCATCACCAC, cav3Fwd CAACGAAGATGTCGTGAAGG, cav3Rvs GAGACGGTGAAGGTGGTG TAA, and for cavin $1 b$ and cavy from ${ }^{17}$.

Electron microscopy. Hearts were fixed in $1 \%$ glutaraldehyde $/ 4 \%$ paraformaldehyde in PBS overnight. Samples were post-fixed in $1 \%$ osmium tetroxide for $60 \mathrm{~min}$ and dehydrated through a series of ethanol solutions $(30 \%, 50 \%, 70 \%, 95 \%, 100 \%)$ and acetone. After the last dehydration step, samples were incubated in a 1:3, 1:1, 3:1 mixture of DURCUPAN resin and acetone and cured at $60^{\circ} \mathrm{C}$ for $48 \mathrm{~h}$. Ultrathin Sections $(50-60 \mathrm{~nm})$ were obtained using a diamond knife (Diatome AG, Biel, Switzerland) in an ultramicrotome (Leica Reichert ultracut S. Leica Microsystems) and collected in 200-mesh copper grids. The sections were counterstained with $2 \%$ uranyl acetate in water for $20 \mathrm{~min}$ followed by a lead citrate solution. Sections were examined with a JEOL JEM1010 electron microscope (Tokyo, Japan) equipped with an Orius SC200 digital camera (Gatan Inc., Pleasanton, CA).

Image analysis and quantification. To analyse cardiomyocyte proliferation, MEF2-positive nuclei were counted in an area of $100 \mu \mathrm{m}$ around the injury site using Fiji (ImageJ, NIH). BrdU-MEF2-positive cells were also counted and the \% proliferation index was expressed as the MEF2:BrdU-MEF2 ratio. For TGF $\beta$ signalling activation, all phospho-smad3-positive cardiomyocyte (100 $\mu \mathrm{m}$ of the injury) or GFP-positive cells (inside the injured area) were counted and normalised to the total number of cardiomyocytes or GFP-positive cells. To quantify the regeneration process, at least three sections, in the middle of the ventricle that contain both of the valves, of each heart were used and the injured area (fibrotic tissue and collagen) was measured using Fiji and expressed as a percentage of the total ventricular area. The $3 \mathrm{D}$ analysis of the whole-mount hearts was carried out using Fiji and IMARIS programmes. The volume of the GFP signal inside the injured area (RFP negative) was also measured and presented in relation to the volume of the injury. Electron microscopy images of the plasma membrane of coronary endothelial cells were taken at 50,000 $\times$ magnification. Uncoated membrane invaginations of $40-90 \mathrm{~nm}$ size were counted ${ }^{17}$ and expressed as density per $\mu \mathrm{m}^{2}$ of the perinuclear area. Two endothelial cells per three sections of the same heart were examined. Fiji was used to calculate the perinuclear area and for caveolae identification.

Atomic force microscopy (AFM)-force spectroscopy. Adult zebrafish were sacrificed by immersion in $0.16 \%$ tricaine and the heart was dissected. The atrium was removed and the ventricle was placed horizontally atop a $4 \%$ agarose gel immersed in PBS with $0.1 \mathrm{M} \mathrm{KCl}$ to arrest the heartbeat uniformly. AFM-force spectroscopy experiments were performed with a JPK Nanowizard III microscope (JPK Instruments, Berlin, Germany) coupled with an inverted optical microscope (AXIO Observer D1; Carl Zeiss, Germany) and equipped with Plateau-CONT-SPL cantilevers (Nanosensors, Neuchatel, Switzerland), with a nominal spring constant of $0.02-0.77 \mathrm{~N} / \mathrm{m}$ and a spherical tip shape $(\mathrm{R}=30 \mu \mathrm{m})$. The actual spring constant of the cantilever was determined using the thermal noise method as implemented in the AFM software. Force-distance curves (FDC) were acquired to determine Young's modulus of the zebrafish heart apex. The tip-sample distance was modulated by applying a triangular waveform ${ }^{16}$. The tip velocity was set to $10 \mu \mathrm{m} / \mathrm{s}$ and the amplitude to $15 \mu \mathrm{m}$. The maximum force exerted on the heart apex during a single FDC was of $11 \mathrm{nN}$. For each zebrafish heart, a complete sequence of 375 FDC was performed. These FDC were distributed in three different areas of $100 \times 100 \mu \mathrm{m}^{2}$ several hundreds of microns apart. In each area, 125 FDC were measured (all along the zebrafish apex). To determine the contact point, we used a ratio of variances protocol. Young's modulus was obtained by fitting a section of the force-distance curve (approach semi-cycle of the whole FDC) with a Hertz model for spherical indenters.

Statistical analysis. Sample sizes, statistical tests and P-values are specified in the figure legends and were determined with GraphPad Prism software (GraphPad Software Inc., San Diego, CA). Statistical tests were twotailed. $P$ values below 0.05 were considered of statistical significance.

Received: 3 March 2020; Accepted: 5 June 2020

Published online: 30 July 2020 


\section{References}

1. Cohen, A. W. et al. Caveolin-1 null mice develop cardiac hypertrophy with hyperactivation of p42/44 MAP kinase in cardiac fibroblasts. Am. J. Physiol. Cell Physiol. 284, C457-474 (2003).

2. Patel, H. H. et al. Mechanisms of cardiac protection from ischemia/reperfusion injury: a role for caveolae and caveolin-1. FASEB J. 21, 1565-1574 (2007).

3. Robenek, H., Weissen-Plenz, G. \& Severs, N. J. Freeze-fracture replica immunolabelling reveals caveolin-1 in the human cardiomyocyte plasma membrane. J. Cell Mol. Med. 12, 2519-2521 (2008).

4. Scherer, P. E. et al. Cell-type and tissue-specific expression of caveolin-2. Caveolins 1 and 2 co-localize and form a stable heterooligomeric complex in vivo. J. Biol. Chem. 272, 29337-29346 (1997).

5. Fra, A. M., Williamson, E., Simons, K. \& De Parton, R. G. novo formation of caveolae in lymphocytes by expression of VIP21caveolin. Proc. Natl. Acad. Sci. USA 92, 8655-8659 (1995).

6. Drab, M. et al. Loss of caveolae, vascular dysfunction, and pulmonary defects in caveolin-1 gene-disrupted mice. Science 293, 2449-2452 (2001).

7. Razani, B. et al. Caveolin-1 null mice are viable but show evidence of hyperproliferative and vascular abnormalities. J. Biol. Chem. 276, 38121-38138 (2001).

8. Zhao, Y. Y. et al. Defects in caveolin-1 cause dilated cardiomyopathy and pulmonary hypertension in knockout mice. Proc. Natl. Acad. Sci. USA 99, 11375-11380 (2002).

9. Liu, L. et al. Deletion of Cavin/PTRF causes global loss of caveolae, dyslipidemia, and glucose intolerance. Cell. Metab. 8, 310-317 (2008).

10. Cheng, J. P. et al. Caveolae protect endothelial cells from membrane rupture during increased cardiac output. J. Cell Biol. 211, 53-61 (2015).

11. Kim, C. A. et al. Association of a homozygous nonsense caveolin-1 mutation with Berardinelli-Seip congenital lipodystrophy. J. Clin. Endocrinol. Metab. 93, 1129-1134 (2008).

12. Razani, B. et al. Caveolin-1 regulates transforming growth factor (TGF)-beta/SMAD signaling through an interaction with the TGF-beta type I receptor. J. Biol. Chem. 276, 6727-6738 (2001).

13. Gervasio, O. L., Phillips, W. D., Cole, L. \& Allen, D. G. Caveolae respond to cell stretch and contribute to stretch-induced signaling. J. Cell Sci. 124, 3581-3590 (2011).

14. Sinha, B. et al. Cells respond to mechanical stress by rapid disassembly of caveolae. Cell 144, 402-413 (2011).

15. Lo, H. P. et al. The caveolin-cavin system plays a conserved and critical role in mechanoprotection of skeletal muscle. J. Cell Biol. 210, 833-849 (2015).

16. Garcia, P. D., Guerrero, C. R. \& Garcia, R. Time-resolved nanomechanics of a single cell under the depolymerization of the cytoskeleton. Nanoscale. 9, 12051-12059 (2017).

17. Lim, Y. W. et al. Caveolae protect notochord cells against catastrophic mechanical failure during development. Curr. Biol. 27, 1968-1981 (2017).

18. Park, D. S. et al. Caveolin-1 null (-/-) mice show dramatic reductions in life span. Biochemistry 42, 15124-15131 (2003).

19. Wunderlich, C. et al. Disruption of caveolin-1 leads to enhanced nitrosative stress and severe systolic and diastolic heart failure. Biochem. Biophys. Res. Commun. 340, 702-708 (2006).

20. Jasmin, J. F. et al. Caveolin-1 deficiency exacerbates cardiac dysfunction and reduces survival in mice with myocardial infarction. Am. J. Physiol. Heart Circ. Physiol. 300, H1274-1281 (2011).

21. Shivshankar, P. et al. Caveolin-1 deletion exacerbates cardiac interstitial fibrosis by promoting M2 macrophage activation in mice after myocardial infarction. J. Mol. Cell Cardiol. 76, 84-93 (2014).

22. Miyasato, S. K. et al. Caveolin-1 modulates TGF-betal signaling in cardiac remodeling. Matrix Biol. 30, 318-329 (2011).

23. Fang, P. K. et al. Caveolin-1alpha and -1beta perform nonredundant roles in early vertebrate development. Am. J. Pathol. 169, 2209-2222 (2006).

24. Cao, J. et al. Single epicardial cell transcriptome sequencing identifies Caveolin 1 as an essential factor in zebrafish heart regeneration. Development. 143, 232-243 (2016).

25. Dufrene, Y. F. et al. Imaging modes of atomic force microscopy for application in molecular and cell biology. Nat. Nanotechnol. 12, 295-307 (2017).

26. Lawson, N. D. \& Weinstein, B. M. In vivo imaging of embryonic vascular development using transgenic zebrafish. Dev. Biol. 248, 307-318 (2002).

27. Gonzalez-Rosa, J. M., Peralta, M. \& Mercader, N. Pan-epicardial lineage tracing reveals that epicardium derived cells give rise to myofibroblasts and perivascular cells during zebrafish heart regeneration. Dev. Biol. 370, 173-186 (2012).

28. Gonzalez-Rosa, J. M. \& Mercader, N. Cryoinjury as a myocardial infarction model for the study of cardiac regeneration in the zebrafish. Nat. Protoc. 7, 782-788 (2012).

29. Kikuchi, K. et al. Retinoic acid production by endocardium and epicardium is an injury response essential for zebrafish heart regeneration. Dev. Cell. 20, 397-404 (2011).

30. Marin-Juez, R. et al. Fast revascularization of the injured area is essential to support zebrafish heart regeneration. Proc. Natl. Acad. Sci. USA 113, 11237-11242 (2016).

31. Munch, J., Grivas, D., Gonzalez-Rajal, A., Torregrosa-Carrion, R. \& de la Pompa, J. L. Notch signalling restricts inflammation and serpinel expression in the dynamic endocardium of the regenerating zebrafish heart. Development. 144, 1425-1440 (2017).

32. Hill, M. M. et al. PTRF-Cavin, a conserved cytoplasmic protein required for caveola formation and function. Cell 132, 113-124 (2008).

33. Hansen, C. G., Shvets, E., Howard, G., Riento, K. \& Nichols, B. J. Deletion of cavin genes reveals tissue-specific mechanisms for morphogenesis of endothelial caveolae. Nat. Commun. 4, 1831 (2013).

34. Chablais, F. \& Jazwinska, A. Induction of myocardial infarction in adult zebrafish using cryoinjury. J. Vis. Exp. 62, e3666 (2012).

35. Gonzalez-Rosa, J. M., Martin, V., Peralta, M., Torres, M. \& Mercader, N. Extensive scar formation and regression during heart regeneration after cryoinjury in zebrafish. Development. 138, 1663-1674 (2011).

36. Schnabel, K., Wu, C. C., Kurth, T. \& Weidinger, G. Regeneration of cryoinjury induced necrotic heart lesions in zebrafish is associated with epicardial activation and cardiomyocyte proliferation. PLoS ONE 6, e18503 (2011).

37. Chablais, F. \& Jazwinska, A. The regenerative capacity of the zebrafish heart is dependent on TGFbeta signaling. Development 139, 1921-1930 (2012).

38. Murata, T. et al. Reexpression of caveolin-1 in endothelium rescues the vascular, cardiac, and pulmonary defects in global caveolin-1 knockout mice. J. Exp. Med. 204, 2373-2382 (2007).

39. Bednarek, D. et al. Telomerase is essential for zebrafish heart regeneration. Cell Rep. 12, 1691-1703 (2015).

40. Mathur, A. B., Collinsworth, A. M., Reichert, W. M., Kraus, W. E. \& Truskey, G. A. Endothelial, cardiac muscle and skeletal muscle exhibit different viscous and elastic properties as determined by atomic force microscopy. J. Biomech. 34, 1545-1553 (2001).

41. Garcia, P. D. \& Garcia, R. Determination of the elastic moduli of a single cell cultured on a rigid support by force microscopy. Biophys. J. 114, 2923-2932 (2018).

42. Poss, K. D., Wilson, L. G. \& Keating, M. T. Heart regeneration in zebrafish. Science 298, 2188-2190 (2002)

43. El-Brolosy, M. A. et al. Genetic compensation triggered by mutant mRNA degradation. Nature 568, 193-197 (2019). 
44. Sanchez-Iranzo, H. et al. Tbx5a lineage tracing shows cardiomyocyte plasticity during zebrafish heart regeneration. Nat. Commun. 9, 428 (2018).

45. Sanz-Morejon, A. et al. Wilms tumor $1 \mathrm{~b}$ expression defines a pro-regenerative macrophage subtype and is required for organ regeneration in the zebrafish. Cell Rep. 28, 1296-1306 (2019).

46. Gosens, R. et al. Caveolin-1 is required for contractile phenotype expression by airway smooth muscle cells. J. Cell. Mol. Med. 15, 2430-2442 (2011).

47. Halayko, A. J., Tran, T. \& Gosens, R. Phenotype and functional plasticity of airway smooth muscle: role of caveolae and caveolins. Proc. Am. Thorac. Soc. 5, 80-88 (2008).

48. Budnar, S. et al. Anillin promotes cell contractility by cyclic resetting of RhoA residence kinetics. Dev Cell. 49, 894-906 (2019).

49. Grande-Garcia, A. et al. Caveolin-1 regulates cell polarization and directional migration through Src kinase and Rho GTPases. J. Cell Biol. 177, 683-694 (2007).

50. Peng, F. et al. RhoA activation in mesangial cells by mechanical strain depends on caveolae and caveolin-1 interaction. J. Am. Soc. Nephrol. 18, 189-198 (2007).

51. Kimmel, C. B., Ballard, W. W., Kimmel, S. R., Ullmann, B. \& Schilling, T. F. Stages of embryonic development of the zebrafish. Dev. Dyn. 203, 253-310 (1995).

52. Cong, L. et al. Multiplex genome engineering using CRISPR/Cas systems. Science 339, 819-823 (2013).

53. Gonzalez-Rosa, J. M. et al. Use of echocardiography reveals reestablishment of ventricular pumping efficiency and partial ventricular wall motion recovery upon ventricular cryoinjury in the zebrafish. PLoS ONE 9, el15604 (2014).

54. Susaki, E. A. et al. Whole-brain imaging with single-cell resolution using chemical cocktails and computational analysis. Cell 157, 726-739 (2014).

55. McCurley, A. T. \& Callard, G. V. Characterization of housekeeping genes in zebrafish: male-female differences and effects of tissue type, developmental stage and chemical treatment. BMC Mol. Biol. 9, 102 (2008).

\section{Acknowledgements}

We thank E. Diaz at the CNIC animal facility for fish husbandry; B. Rios, V. García, L. Méndez for technical support; the CNIC Microscopy Unit for help; A.Vanesa Alonso and L. Flores for support with the echocardiographic experiment; F. Urbano and C. Aguado for support and help with the TEM. This work was supported by Grants SAF2016-78370-R, CB16/11/00399 (CIBER CV) and RD16/0011/0021 (TERCEL) from the Spanish Ministry of Science, Innovation and Universities (MCNU) and Grants from the Fundación BBVA (Ref.: BIO14_298), Fundación La Marató (Ref.: 20153431) and CardioNeT (Ref.: 28600) from the European Commission to J.L.d.l.P. Grants MAT2016-76507-R (MCIU), Comunidad de Madrid (Ref. S2018/NMT-4443), Tec4Bio and European Research Council (ERC-AdG-340177) were awarded to R. G. D.G. held a PhD fellowship linked to Grant CardioNeT. The cost of this publication was supported in part with funds from the European Regional Development Fund. The CNIC is supported by the Instituto de Salud Carlos III (ISCIII), the MCNU and the Pro CNIC Foundation, and is a Severo Ochoa Centre of Excellence (SEV-2015-0505).

\section{Author contributions}

D.G., and A.G.-R. performed experiments, C.G.R. and R.G. performed and analysed AFM experiments. D.G. and J.L.d.l.P. designed experiments, reviewed all the data, prepared figures and wrote the manuscript. All authors reviewed the manuscript during its preparation.

\section{Competing interests}

The authors declare no competing interests.

\section{Additional information}

Supplementary information is available for this paper at https://doi.org/10.1038/s41598-020-68802-9.

Correspondence and requests for materials should be addressed to J.L.d.l.P.

Reprints and permissions information is available at www.nature.com/reprints.

Publisher's note Springer Nature remains neutral with regard to jurisdictional claims in published maps and institutional affiliations.

\footnotetext{
(c) (i) Open Access This article is licensed under a Creative Commons Attribution 4.0 International License, which permits use, sharing, adaptation, distribution and reproduction in any medium or format, as long as you give appropriate credit to the original author(s) and the source, provide a link to the Creative Commons license, and indicate if changes were made. The images or other third party material in this article are included in the article's Creative Commons license, unless indicated otherwise in a credit line to the material. If material is not included in the article's Creative Commons license and your intended use is not permitted by statutory regulation or exceeds the permitted use, you will need to obtain permission directly from the copyright holder. To view a copy of this license, visit http://creativecommons.org/licenses/by/4.0/.
}

(C) The Author(s) 2020 